\title{
Implementation of music equalization simulink model on DSK6713
}

\author{
Deepali Yoginath Loni \\ Department of Electronics, D.KT.Es' Textile \& Engineering Institute, Ichalkaranji, India
}

Email address:

deepaliloni@rediffmail.com

\section{To cite this article:}

Deepali Yoginath Loni. Implementation of Music Equalization Simulink Model on DSK6713. Science Journal of Circuits, Systems and Signal Processing. Vol. 2, No. 2, 2013, pp. 74-77. doi: 10.11648/j.cssp.20130202.16

\begin{abstract}
Audio equalization is a technique which consists of boosting or cutting certain frequency components of a given signal for sound quality enhancement. Equalizer is an electronic device or type of software that increases and decreases the power of sound waves. The paper deals with the analysis of music signals to develop insight on its frequency bands and auditory perception, through a case study of audio equalization. The graphic equalizer is designed through a Simulink model and is implemented in TMS320C6713 DSP board. This project is developed in order to deliver an insight on how audio equalization can be implemented with Matlab / Simulink for educational purposes.
\end{abstract}

Keywords: Equalization, Simulink, Gain, DSP, TMS320C6713

\section{Introduction}

Music equalizers are devices or software used for amplifying and attenuating predetermined frequency bands. In many fields of sound processing like sound recording studios, or sound transmission to the listener during a concert, equalizers are applied for sound control and enhancement. Although initially developed for sound recording studios, equalizers have rapidly gained acceptance and can actually be found in almost every consumer product like car radios, hifi-amplifiers, or MP3 players.

Equalizers can be classified into two main groups: graphic and parametric equalizers. While parametric equalizers have their name from the fact that parameters of the filters are fully adjustable, graphic equalizers get their name from the fact that the relative positions of the sliders build a graphic picture of the desired magnitude response [1]. For graphic equalizers we only control with sliders the boost or cut by keeping the center or middle frequency unchanged. A graphic equalizer is a set of band-pass filters each with fixed center frequency and a certain bandwidth, where the first and last bands are respectively low-/high pass filters. In this paper, we will focus on a graphic equalizer design. The model will cover a frequency range up to $16 \mathrm{kHz}$ with a gain adjustment for each frequency band. The remainder of this paper is organized as follows. In Section 2, we will introduce the proposed system for implementing the equalizer on DSP board. In Section 3 the Simulink model with its design parameters are explained. Section 4 describes the procedure to run the Simulink model in real-time using a TMS320C6713 DSP board. In Section 5 and 6 results and conclusion are mentioned.

\section{The Proposed System: Implementing Music Equalizer on DSK6713 Processor}

The system in Fig. 1 is an audio equalizer that typically adjusts the energy levels of the audio data in one or more different frequency bands in order to change the characteristics of the audio data. This equalizer allows boosting (or suppression/attenuation) of frequencies between the source of a sound and the output of the sound.

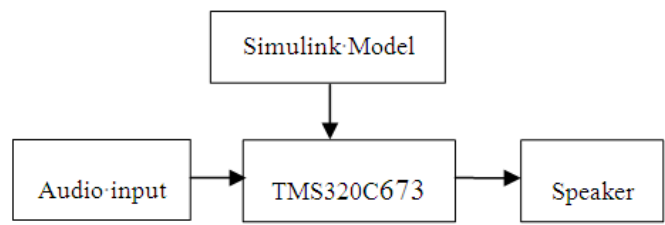

Figure 1. System implementing music equalizer using TMS320C6713.

The equalizer system of Fig. 1 has two major parts, the DSP Processor and the Simulink model. 


\subsection{TMS320C6713 DSK}

The TMS320C6713 device is based on the high-performance advanced Velocity very - long instruction-word (VLIW) architecture developed by Texas Instruments (TI), making this DSP an excellent choice for multichannel and multifunction applications [2]. It provides an efficient and stable DSP development environment and is a robust, low-cost and easily available DSK in both universities and industry [3].

The audio input is applied to Line-in of DSK which is taken either from a PC or through a mobile. Mono channel is used for simplicity. The sampling frequency is set to 48 $\mathrm{kHz}$. Word length is 16-bit, scaling is normalized and samples per frame are 64. Simulink model for equalization are built with the help of Simulink blocksets and its equivalent ' $C$ ' code is transferred to the DSP Starter Kit with the help of USB cable. Actual signal processing is done in DSK with the help of ' $C$ ' code downloaded in it which generates an equalized music output. Output is taken from the 'Headphone out' of the DSK and is applied to speaker.

\subsection{Simulink}

Simulink is a tool for simulating dynamic systems with a graphical interface specially developed for this purpose. Within the Matlab environment, Simulink is a Matlab toolbox that differs from the other toolboxes, both in this special interface and in the special "programming technique" associated with it. There are many advantages in programming DSP algorithms using Matlab. These include ease of coding, able to use a powerful set of inbuilt functions and seamless link between Matlab and Simulink [4].

Simulink is an attempt to understand the behavior of the system by means of a graphical representation, which essentially consists of representations of individual components of the system together with the signal flow between these components.

\subsubsection{Advantages of Simulink}

- One can easily build any dynamic, linear and non linear systems with the help of Simulink blocksets.

- Build closed loop control systems, signal routing and user defined subsystems with the help of Simulink.

- When interfacing Simulink with DSK, the Code Composer Studio (CCS) automatically generates 'C' code of the model.

- Code generation and simulation is easy.

\section{The Simulink Model of Music Equalization}

Simulink uses graphical block diagrams to create models for real-time implementation of applications and then use Real-Time Workshop to generate $\mathrm{C}$ code targeted to the TI DSP board by means of Code Composer Studio (CCS IDE) [5]. The Simulink model of equalizer is shown in Fig. 2. For downloading this Simulink model into DSK, it is essential to include C6713 DSK Board Support Library Blocks in the model so as to establish a communication with the codec of target C6713 DSK [6 - 9].

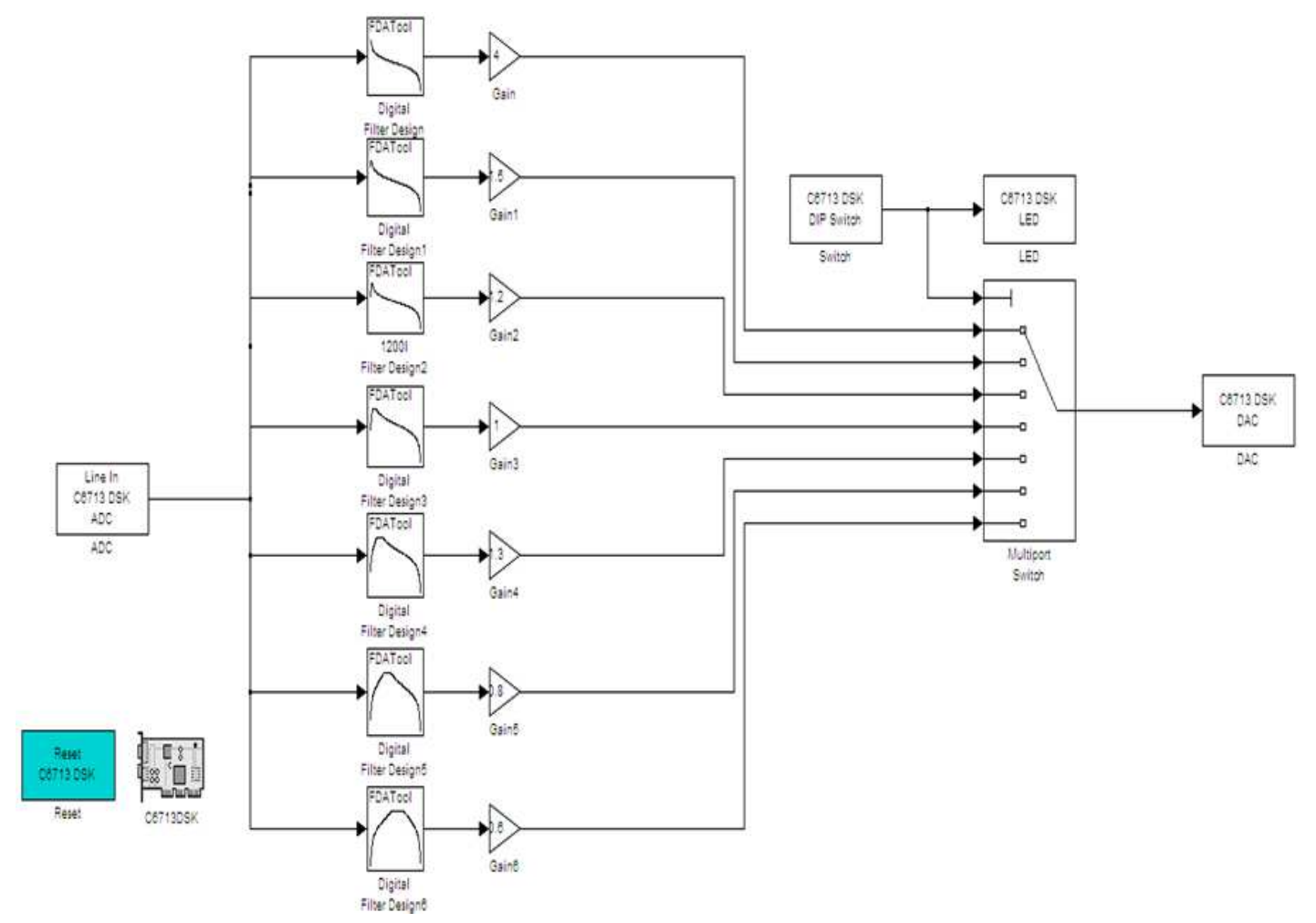

Figure 2. Simulink model of equalization. 
Equalizers normally work on groups of frequencies called frequency bands or more commonly just bands. The Simulink model incorporates filters for performing the task of separating the signal of different frequency bands. One low pass and six band pass filters are designed in Matlab using FDATOOL, for filtering seven different bands of frequencies. IIR filters are preferred as they faster than the FIR filter and give smoother amplitude response. The key to filtering the music signal correctly is to design filters with overlapping cut off frequencies. The cut off frequencies of all filters shown in the model are listed in Table 1.

Table 1. Cutoff frequencies of each filter block in Simulink model

\begin{tabular}{lcc}
\hline Filter type & Lower cutoff frequency(Hz) & $\begin{array}{c}\text { Upper cutoff } \\
\text { frequency(Hz) }\end{array}$ \\
\hline Lowpass & 200 & - \\
Bandpass & 200 & 600 \\
Bandpass & 600 & 1000 \\
Bandpass & 1000 & 3000 \\
Bandpass & 3000 & 6000 \\
Bandpass & 6000 & 10,000 \\
Bandpass & 10,000 & 16,000 \\
\hline
\end{tabular}

The frequency bands cover the full audio range of $20 \mathrm{~Hz}$ to $20 \mathrm{KHz}$. Further to have fine-grained control over the gain (boost) or suppression (attenuation) within the bands, the Simulink model includes a Gain blockset. Gain setting of each frequency band for generating various music effects is shown in Table 2.

Table 2. Gain setting values of each gain block in the model

\begin{tabular}{lc}
\hline Frequency Band & Gain \\
\hline $0-200$ & 4 \\
$200-600$ & 1.5 \\
$600-1000$ & 1.2 \\
$1000-3000$ & 1 \\
$3000-6000$ & 1.3 \\
$6000-10000$ & 0.8 \\
$10000-16000$ & 0.6 \\
\hline
\end{tabular}

DIP switches in the model control the multiport switch operation. As per the DIP switch setting, the multiport switch selects the corresponding filter band and permits its output to be available to the speaker. LEDs on the Kit display the DIP switch value.

\section{Executing Simulink Model on DSK}

Steps involved in building and executing the Simulink model on C6713 DSK:

1. Build the Simulink model using the blocksets from Simulink Library Browser in Matlab r2007a.

2. Open "TIC6000 Target Preferences" from Simulink Library Embedded target Content for TI C6000.
3. Select 6713DSK from the list of DSK's available and add C6713 DSK board support components to the model (like ADC, DAC, RESET, DIP Switch and LED).

4. Run the CCS software in background.

5. Apply the input music signal to the Line In input of DSK and connect the speakers to the Line Out.

6. Open the Simulink model of equalization and press CNTRL + B to build an equivalent ' $\mathrm{C}$ ' language code in CCS. Simulink starts communicating with CCS. The CCS automatically gets open and the .out File will be loaded into the DSP.

7. Select the band by setting the DIP switch and the corresponding equalized sound will be heard through the speaker.

8. To stop model execution, click the Reset DSK block or use the Halt option in CCS.

\section{Results}

The results can be interpreted and analyzed only by subjective listening. The Table 3 indicates the effect observed for the applied music signal corresponding to the setting of DIP switch.

Table 3. Effects observed on music signal

\begin{tabular}{cccc}
\hline $\begin{array}{c}\text { DIP switch } \\
\text { setting }\end{array}$ & $\begin{array}{c}\text { Frequency } \\
\text { Band }\end{array}$ & Gain & Effects \\
\hline $\begin{array}{c}\text { Switch1 } \\
(0001)\end{array}$ & $0-200$ & 4 & $\begin{array}{c}\text { Non-vocal portion of music. } \\
\text { Only background music like } \\
\text { effect of drum is observed. } \\
\text { Non-vocal portion of music. }\end{array}$ \\
$\begin{array}{c}\text { Switch2 } \\
(0010)\end{array}$ & $200-600$ & 1.5 & $\begin{array}{c}\text { Effect of big instruments such } \\
\text { as guitar, violin. }\end{array}$ \\
$\begin{array}{c}\text { Switch3 } \\
(0011)\end{array}$ & $600-1000$ & 1.2 & $\begin{array}{c}\text { Vocal effect is observed. Actual } \\
\text { singer voice comes into picture. }\end{array}$ \\
$\begin{array}{c}\text { Switch4 } \\
(0100) \\
\text { Switch5 } \\
(0101)\end{array}$ & $1000-3000$ & 1 & $\begin{array}{c}\text { Clarity and thinness of vocal is } \\
\text { observed }\end{array}$ \\
$\begin{array}{c}\text { Switch6 } \\
(0110)\end{array}$ & $6000-10,000$ & 0.8 & $\begin{array}{c}\text { Treble mode. Shows small } \\
\text { instruments effect. }\end{array}$ \\
$\begin{array}{c}\text { Switch7 } \\
(0111)\end{array}$ & $10,000-16,000$ & 0.6 & $\begin{array}{c}\text { Treble mode. Shows small } \\
\text { instruments effect. }\end{array}$ \\
\hline
\end{tabular}

\section{Conclusion}

The paper obtain an audio balance where each piece of a musical composition, such as drum beats, guitar riffs and vocals, can be distinctly heard and sound good as a unit. The work can be used for applications such as making sounds more intelligible. For instance, if an instrument sound like a drum is masking out the sound of a guitar or a vocal, the equalizer can increase the strength and resonance of the weaker part. Also the work presents the communication and interface of Simulink model with DSK. 
The work can be further extended for implementing parametric equalizer.

\section{References}

[1] F.X. Nsabimana, U. Zölzer, "Real-Time Implementation of a 1/3-Octave Audio Equalizer Simulink Model using TMS320C6416 DSP Board", EDERS'06, 4. April 2006, Munich, Germany ( Presented as lecture )

[2] Application Report SPRA867 - Parametric Equalization on TMS320C6000 DSP Remi Payan Catalog DSP. December 2002.

[3] T. J. Ahmad, H. Ali, M. A. Ajaz, S. A. Khan, "A DSK Based Simplified Speech Processing Module for Cochlear Implant", IEEE International Conference on Acoustics, Speech and Signal Processing, 2009. http://dx.doi.org/10.1109/ICASSP.2009.495959

[4] W. S. Gan1, S. M. Kuo2, "Transition from Simulink to
MATLAB in Real-Time Digital Signal Processing Education", International Journal Engineering Education, Vol. 21, No. 4, pp. $587 \pm 595,2005$.

[5] J. Zapata, R. Ruiz, "Rapid Development of Real-Time Applications Using MATLAB/Simulink on TI C6000-based DSP", Proceedings of the 5th WSEAS International Conference on Education and Educational Technology, Tenerife, Canary Islands, Spain, December 16- 18, 2006,pp.104-110.

[6] The MathWorks, Signal Processing Toolbox User's Guide, Version 6, 2003.

[7] The MathWorks, DSP Blockset User's Guide, Version 5, 2003.

[8] The MathWorks Embedded Target for the TI TMS320C6000 ${ }^{\mathrm{TM}}$ DSP Platform, Version1, 2002.

[9] Texas Instruments, C6713 DSP Starter Kit. Available at:http://focus.ti.com/docs/toolsw/folders/print/tmds 20006713.html220 